\title{
Bottom-Up Fabrication of PEG Brush on Poly(dimethylsiloxane) for Antifouling Surface Construction
}

\author{
Junmei Tang, Yuemei Han, Hao Chen, and Quankui Lin \\ School of Ophthalmology \& Optometry, Eye Hospital, Wenzhou Medical University, Wenzhou 325027, China \\ Correspondence should be addressed to Quankui Lin; lqk97531@126.com
}

Received 5 May 2016; Accepted 26 May 2016

Academic Editor: Weifeng Zhao

Copyright ( 2016 Junmei Tang et al. This is an open access article distributed under the Creative Commons Attribution License, which permits unrestricted use, distribution, and reproduction in any medium, provided the original work is properly cited.

Poly(dimethylsiloxane) silicones have found many applications in biomedical devices, whereas their surface hydrophobicity always brings about unexpected bioadhesion, causing complications of the implanted biomedical devices. In this work, surface-initiated reversible addition-fragmentation chain transfer (SI-RAFT) polymerization was utilized to generate PEG brushes on silicone surface, obtaining highly hydrophilic surface coatings. Such PEG brush coated silicone presents excellent antifouling to protein, cells, and bacteria, which may have great potential in implantable biomaterial surface modifications.

\section{Introduction}

Poly(dimethylsiloxane) (PDMS) silicones have found many applications in biomedical devices, such as catheters, prostheses, contact lens, and intraocular lens [1]. But their hydrophobicity always brings about unexpected bioadhesion on the surface, which may cause the main complications of the implanted biomedical devices. For example, the adhesion and proliferation of the lens epithelial cells on the silicone intraocular lens may cause the after cataract, a common complication after the cataract surgery, which leads to losing vision again [2]. It is also common that the rapid accumulation of tear proteins or the adhesion of bacteria may potentially cause the adverse clinical events in contact lens wearing [3]. These complications are related to the biofouling of the silicone materials.

Generally, biofouling is generated by the attachment of microorganisms such as cells or bacteria to the surface. To circumvent such problems, a variety of strategies have been developed to modify the surface properties of PDMS by physical modification or chemical covalent methods, including oxygen plasma, ultraviolet light/ozone, polyelectrolyte multilayers, surface activation, and chemical grafting [4-8]. Particularly, polyethylene glycol (PEG) surface modification is an effective method for antifouling purpose $[7,9]$. The PEG with active ending groups can be coupled to the surface via chemical grafting. However, the
PEGylated surface fabricated from "graft-to" method only renders short term effects on antifouling, which may be due to the sparseness and irregular arrangement of the PEG chains $[10,11]$. The favorable antifouling surface calls for the well-defined PEG coating techniques.

Surface-initiated living radical polymerization reactions provide a good alternative for the biomedical materials surface modification, as obtaining the well-defined polymer brushes. Reversible addition-fragmentation chain transfer (RAFT) polymerization is one of the living radical polymerization techniques and it has enjoyed widespread acceptance as a modification technique due to its capability to control polymerization of diverse monomers under mild reaction conditions without the requirement of metal catalysts [12-14]. Antifouling surface can be obtained via surfaceinitiated RAFT polymerization of antifouling molecules on the material surface [15-17]. In this work, surface-initiated RAFT (SI-RAFT) was carried out for bottom-up grafting PEG brushes on silicone surface for antifouling applications. The hydrophilicity of the coatings was investigated and the antifouling properties were tested by protein adsorption, $S$. aureus adhesion, and lens epithelial cell adhesion assays.

\section{Materials and Methods}

2.1. Materials. PDMS precursors Sylgard 184 were purchased from Dow Corning Corporation, USA. 


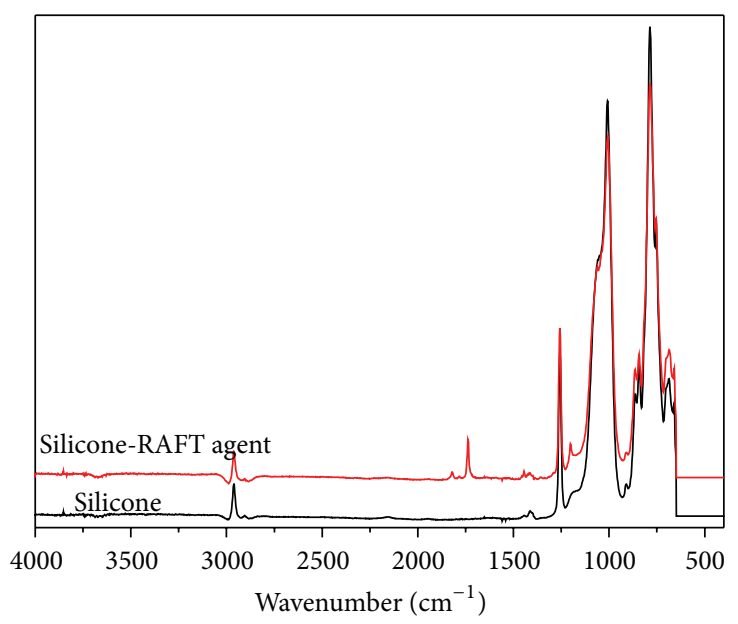

(a)

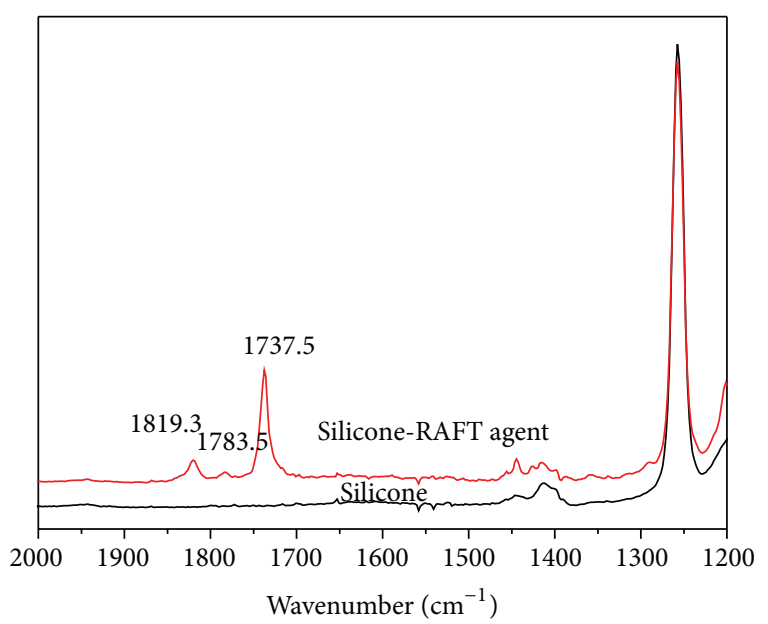

(b)

FIgURE 1: (a) The ATR-FTIR spectra of the silicone before and after RAFT agent immobilization. (b) The amplification of the ATR-FTIR spectra in the wavenumber range of 1200 to $2000 \mathrm{~cm}^{-1}$.

(3-Aminopropyl)triethoxysilane (APTES), 4-cyano-4-(phenylcarbonothioylthio)pentanoic acid (CPCTTPA), N-(3dimethylaminopropyl)- $\mathrm{N}^{\prime}$-ethylcarbodiimide hydrochloride (EDC), N-hydroxysulfosuccinimide sodium (NHS), polyethylene glycol methacrylate (PEGMA, Mn = 360), and 4-cyano-4-(2-cyano-5-hydroxy-5-oxopentan-2-yl) diazenylpentanoic acid (V501) were purchased from Sigma, USA.

2.2. Experimental Methods. Silicone was made from PDMS at a weight ratio of $\mathrm{A}: \mathrm{B}=1: 10$, according to the supplier. The surface modification is initiated by the RAFT agent immobilization onto the silicone. Briefly, silicone was immerged in APTES/ethanol (1\%, w/v) solution overnight. The aminolyzed silicone was then immersed into a reaction vessel containing 4-cyano-4-(phenylcarbonothioylthio)pentanoic acid (CPCTTPA, $0.22 \mathrm{mg} / \mathrm{mL}$ ), $\mathrm{N}$-(3-dimethylaminopropyl)- $\mathrm{N}^{\prime}$-ethylcarbodiimide hydrochloride (EDC, $40 \mathrm{mg} / \mathrm{mL}$ ), and N-hydroxysulfosuccinimide sodium (NHS, $20 \mathrm{mg} / \mathrm{mL}$ ) in distilled water. The reaction mixture was left on a shaking device for $48 \mathrm{~h}$ at room temperature. The CPCTTPA immobilized silicone was used for SI-RAFT polymerization. Typically, polyethylene glycol methacrylate (PEGMA, $\mathrm{Mn}=360$ ) and $0.5 \mathrm{mg} / \mathrm{mL} 4$-cyano-4-(2-cyano5-hydroxy-5-oxopentan-2-yl) diazenylpentanoic acid (V501) were dissolved with ethanol in microwave-safe glass vials and degassed. The vials were placed in Initiator Microwave System (Initiator-60, Biotage, Sweden) to perform polymerization at $60^{\circ} \mathrm{C}$ for predetermined time. After reaction, the functionalized silicone was removed from the solution, washed with ethanol and distilled water ultrasonically, and dried under vacuum overnight. Fourier transform infrared (FTIR) spectroscopy in attenuated total reflection (ATR) mode was used to characterize the immobilization of the RAFT agent and contact angle observation was utilized to evaluate the hydrophilicity of PEG brush modified silicone surface. The protein adsorption, S. aureus adhesion, and lens epithelial cell adhesion assays were then carried out on the functionalized silicone substrate according to the procedures in our previous publications $[5,6,18,19]$.

\section{Results and Discussion}

Figure 1 shows the ATR-FTIR curves of the silicone substrate before or after RAFT agent immobilization. As it can be seen in both of the spectra, there is a strongest band at the wavenumbers from 1000 to $1120 \mathrm{~cm}^{-1}$, which are attributed to the stretching vibration peaks of Si-O-Si. The asymmetric and symmetric stretching vibrations of $\mathrm{C}-\mathrm{H}_{3}$ occur at 2962 and $2909 \mathrm{~cm}^{-1}$, respectively. A sharp single peak is seen in every spectrum at $1250 \mathrm{~cm}^{-1}$, attributed to the deformation vibration of $\mathrm{C}-\mathrm{H}_{3}$ in $\mathrm{Si}-\mathrm{Me}_{2}$ group [20]. Compared to the general symmetric deformation vibration of $\mathrm{C}-\mathrm{H}_{3}$ in alkyl group, this vibration band takes a distinct red shift from 1375 to $1250 \mathrm{~cm}^{-1}$. There is a tiny peak on the silicone spectra at $2160 \mathrm{~cm}^{-1}$ and it disappears after the RAFT agent immobilization. The characteristic vibration of $2160 \mathrm{~cm}^{-1}$ is conventionally attributed to the stretching vibration of $\mathrm{Si}-\mathrm{H}$ bond. The Si-H should disappear after the surface reacted with the silane coupling agent as the $\mathrm{Si}-\mathrm{H}$ was substituted by the $\mathrm{Si}-$ $\mathrm{O}-\mathrm{Si}$ after reaction. There are also some new characteristic peaks which appear after the RAFT agent immobilization. As shown in Figure 1(b), the peak at $1737.5 \mathrm{~cm}^{-1}$ is due to the characteristic absorption bands of the carbonyl group, originated from the carboxyl group in the RAFT agent. Peak at $1443.2 \mathrm{~cm}^{-1}$ is also originated from the vibration of S$\mathrm{C}$ in the RAFT agent. These results indicate the successful immobilization of the RAFT agent on the silicone surface.

Figure 2 shows the results of surface contact angle (SCA) measurements of the silicone before and after SI-RAFT polymerization of PEGMA. The silicone is a material with high hydrophobic surface properties. The initial value of SCA is measured after the water drop contacting with the surface for $30 \mathrm{~s}$. The initial SCA of silicone is $112.8^{\circ}$ (Figure 2(a)(a1)). 


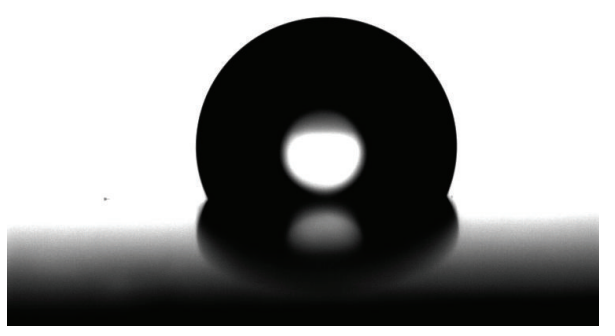

(a1)

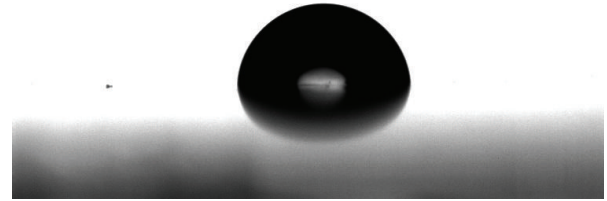

(a2)

(a)

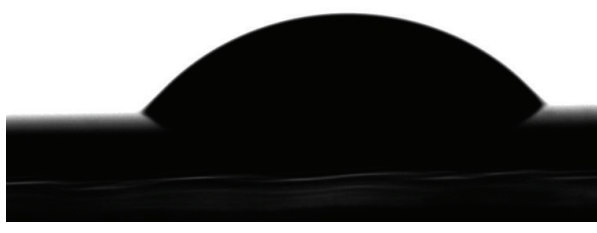

(b1)

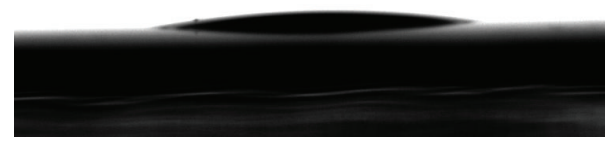

(b2)

(b)

FIGURE 2: Representative surface contact angle images of silicone with (b) or without (a) PEG brush modification. The images were obtained after water drops contacting with the surface for $30 \mathrm{~s}(\mathrm{a} 1, \mathrm{~b} 1)$ and $11 \mathrm{~min}(\mathrm{a} 2, \mathrm{~b} 2)$.

After surface modification of PEG brushes via SI-RAFT, the SCA decreases to $50.6^{\circ}$ (Figure 2(b)(b1)). The surface hydrophilicity is greatly improved after the PEG brushes surface modification. More interestingly, the SCA of the PEG brushes modified surface decreases to less than $8.0^{\circ}$ after the water drop contacting with the surface for $11 \mathrm{~min}$ (Figure 2(b)(b2)), whereas the SCA of pristine silicone is $84.2^{\circ}$ (Figure 2(a)(a2)). The silicone is a hydrophobic material; the small decline of the SCA on silicone surface with time increase may be due to the water evaporation. However, the dramatic decrease of the SCA on the PEG brushes modified surface is not just due to the evaporation. The SI-RAFT polymerization on the surface can obtain a brush structured polymer on the surface $[15,16]$. The brush structure plays an important role in high hydrophilicity of the PEG modified surface. Surface with excellent hydrophilicity is obtained after the SI-RAFT polymerization of PEG on the surface as PEG brushes are generated via bottom-up living polymerization.

Figure 3 shows the protein adsorption results on the silicone before and after PEG brushes modification. The albumin is used as a modal protein for test. The pristine silicone easily absorbs proteins due to its hydrophobic nature. However, the hydrophilic PEG brushes modification greatly reduces the protein adsorption on the surface. The absorbed protein on the modified surface is about $40 \%$ of that on the pristine surface. Figure 4 shows the cell and bacteria adhesion results on the surface. The lens epithelial cell (LEC) and S. aureus are used. As we can see, the pristine silicone is bioadhesive for both LEC and S. aureus. Plenty of LEC and S. aureus are

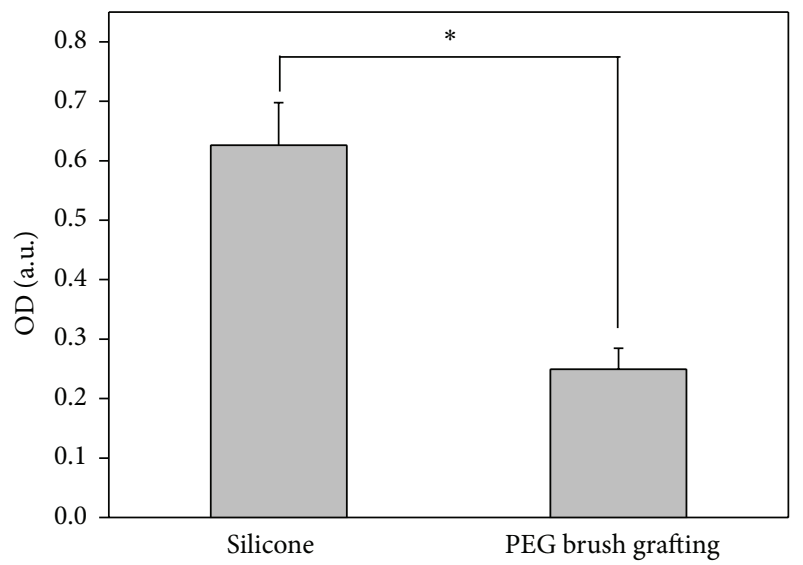

FIGURE 3: The protein adsorption of silicone before and after PEG brush grafting. " Significant difference.

found on the silicone surface (Figures 4(a) and 4(c)). The adhered cells present spreading morphology on the surface, indicating the high cell viability on such surface. The PEG brushes modification greatly reduced the cell and bacteria adhesion. Seldom cells are found on the modified surface. The few adhered cells render small round morphology, which indicates the lack of cell viability on such surface. The cell and bacterial adhesion on the implantable materials may cause lots of complications. For example, the LEC adhesion onto the material surface is the main reason for the silicone intraocular 


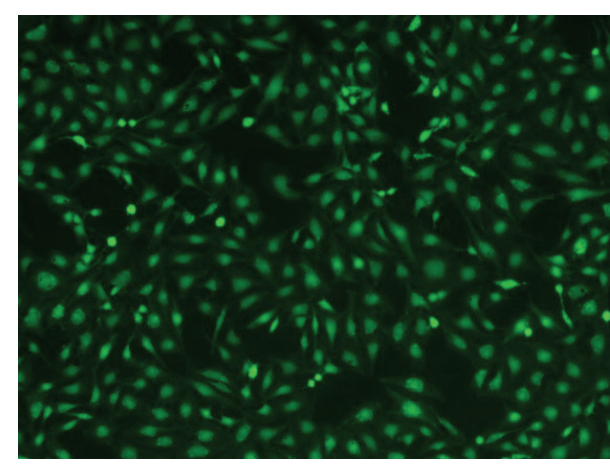

(a)

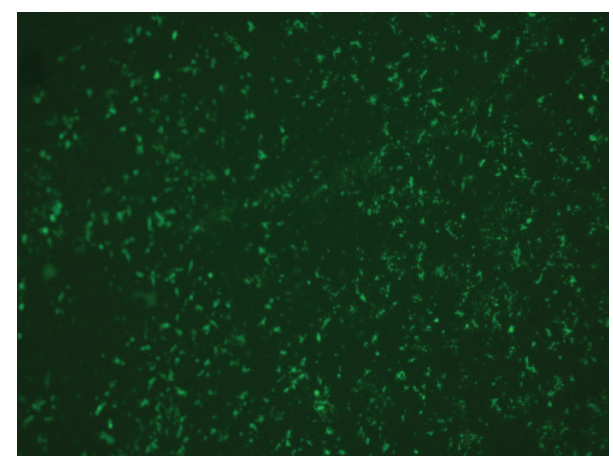

(c)

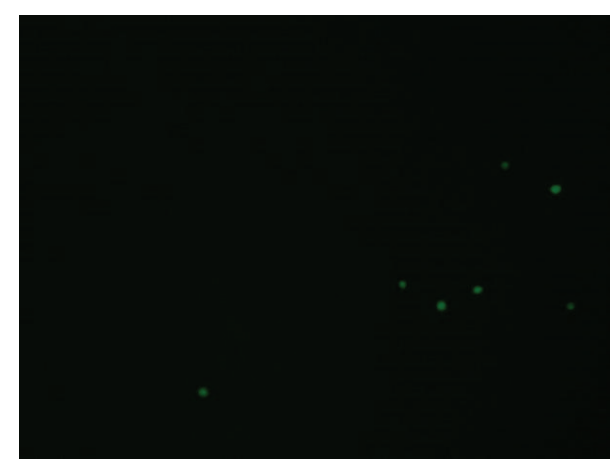

(b)

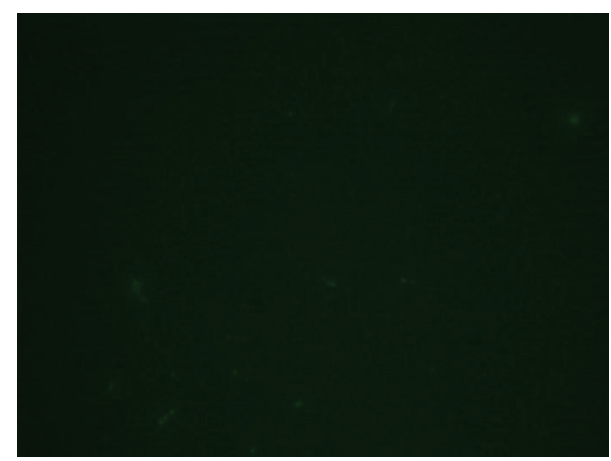

(d)

Figure 4: The lens epithelial cell (a, b) and S. aureus (c, d) adhesion on silicone before and after PEG brush grafting.

lens implantation. S. aureus adhesion and invasion may cause endophthalmitis, which is a critical issue of the vision loss in ophthalmologic surgery. The above result indicates that an excellent antifouling surface is obtained via SIRAFT polymerization of PEGMA on silicone surface, which greatly improves the biocompatibility for biomaterial applications.

\section{Conclusions}

In this paper, a PEG brush coating is obtained via surface-initiated RAFT polymerization of polyethylene glycol methacrylate on silicone surface. The surface contact angle analysis shows the excellent hydrophilicity of the PEG brush coating surface. The in vitro protein adsorption assay indicates that the PEG brush coating can notably decrease the protein adsorption on the surface. The lens epithelial cell and $S$. aureus adhesion assay results indicate that such PEG brush modification can evidently resist the cell and bacteria adhesion. Such antifouling silicone with excellent hydrophilic PEG brush coatings might have great potential in the implantable biomaterials.

\section{Competing Interests}

The authors declare that there are no competing interests regarding the publication of this paper.

\section{Authors' Contributions}

Junmei Tang and Yuemei Han contributed equally to this paper.

\section{Acknowledgments}

The authors greatly acknowledge the financial support from National Natural Science Foundation of China (31570959 and 51203120), Natural Science Foundation of Zhejiang Province (LY16H120003 and LQ12E03001), Medical \& Health Technology Program of Zhejiang Province (2014KYA149), Public Welfare Science \& Technology Program of Wenzhou (Y20150083 and Y20120201), and Xinmiao Talents Program of Zhejiang Province (2015R413074).

\section{References}

[1] M. Li, K. G. Neoh, L. Q. Xu et al., "Surface modification of silicone for biomedical applications requiring long-term antibacterial, antifouling, and hemocompatible properties," Langmuir, vol. 28, no. 47, pp. 16408-16422, 2012.

[2] L. Werner, "Biocompatibility of intraocular lens materials," Current Opinion in Ophthalmology, vol. 19, no. 1, pp. 41-49, 2008.

[3] H. Thissen, T. Gengenbach, R. du Toit et al., "Clinical observations of biofouling on PEO coated silicone hydrogel contact lenses," Biomaterials, vol. 31, no. 21, pp. 5510-5519, 2010. 
[4] W. Mussard, N. Kebir, I. Kriegel, M. Estève, and V. Semetey, "Facile and efficient control of Bioadhesion on poly(dimethylsiloxane) by using a biomimetic approach," Angewandte Chemie-International Edition, vol. 50, no. 46, pp. 10871-10874, 2011.

[5] Q. Lin, X. Xu, B. Wang et al., "Hydrated polysaccharide multilayer as an intraocular lens surface coating for biocompatibility improvements," Journal of Materials Chemistry B, vol. 3, no. 18, pp. 3695-3703, 2015.

[6] B. L. Wang, Q. K. Lin, T. W. Jin et al., "Surface modification of intraocular lenses with hyaluronic acid and lysozyme for the prevention of endophthalmitis and posterior capsule opacification," RSC Advances, vol. 5, no. 5, pp. 3597-3604, 2015.

[7] H. Chen, Y. Chen, H. Sheardown, and M. A. Brook, "Immobilization of heparin on a silicone surface through a heterobifunctional PEG spacer," Biomaterials, vol. 26, no. 35, pp. 7418-7424, 2005.

[8] C. Wetzel, J. Schönfelder, W. Schwarz, and R. H. W. Funk, "Surface modification of polyurethane and silicone for therapeutic medical technics by means of electron beam," Surface \& Coatings Technology, vol. 205, no. 5, pp. 1618-1623, 2010.

[9] K. Chawla, S. Lee, B. P. Lee, J. L. Dalsin, P. B. Messersmith, and N. D. Spencer, "A novel low-friction surface for biomedical applications: modification of poly(dimethylsiloxane) (PDMS) with polyethylene glycol(PEG)-DOPA-lysine," Journal of Biomedical Materials Research Part A, vol. 90, no. 3, pp. 742-749, 2009.

[10] Â. Serrano, O. Sterner, S. Mieszkin et al., "Nonfouling response of hydrophilic uncharged polymers," Advanced Functional Materials, vol. 23, no. 46, pp. 5706-5718, 2013.

[11] X. Xu, J.-M. Tang, Y.-M. Han, W. Wang, H. Chen, and Q.-K. Lin, "Surface PEGylation of intraocular lens for PCO prevention: an in vivo evaluation," Journal of Biomaterials Applications, 2016.

[12] Y. Shi, M. Liu, K. Wang et al., "Direct surface PEGylation of nanodiamond via RAFT polymerization," Applied Surface Science, vol. 357, pp. 2147-2153, 2015.

[13] K. Ohno, Y. Ma, Y. Huang et al., "Surface-initiated reversible addition-fragmentation chain transfer (RAFT) polymerization from fine particles functionalized with trithiocarbonates," Macromolecules, vol. 44, no. 22, pp. 8944-8953, 2011.

[14] M. C. R. Tria, C. D. T. Grande, R. R. Ponnapati, and R. C. Advincula, "Electrochemical deposition and surface-initiated RAFT polymerization: protein and cell-resistant PPEGMEMA polymer brushes," Biomacromolecules, vol. 11, no. 12, pp. 34223431, 2010.

[15] F. Audouin and A. Heise, "Surface-initiated RAFT polymerization of NIPAM from monolithic macroporous polyHIPE," European Polymer Journal, vol. 49, no. 5, pp. 1073-1079, 2013.

[16] J. Yuan, X. Huang, P. Li, L. Li, and J. Shen, "Surface-initiated RAFT polymerization of sulfobetaine from cellulose membranes to improve hemocompatibility and antibiofouling property," Polymer Chemistry, vol. 4, no. 19, pp. 5074-5085, 2013.

[17] B. L. Wang, T. W. Jin, Y. M. Han et al., "Surface-initiated RAFT polymerization of $\mathrm{p}$ (MA POSS-co-DMAEMA ${ }^{+}$) brushes on PDMS for improving antiadhesive and antibacterial properties," International Journal of Polymeric Materials and Polymeric Biomaterials, vol. 65, no. 2, pp. 55-64, 2016.

[18] Q. K. Lin, X. Ding, F. Y. Qiu, X. Song, G. Fu, and J. Ji, "In situ endothelialization of intravascular stents coated with an antiCD34 antibody functionalized heparin-collagen multilayer," Biomaterials, vol. 31, no. 14, pp. 4017-4025, 2010.
[19] Q. K. Lin, X. Xu, Y. Q. Wang et al., "Antiadhesive and antibacterial polysaccharide multilayer as IOL coating for prevention of postoperative infectious endophthalmitis," International Journal of Polymeric Materials and Polymeric Biomaterials, 2016.

[20] D.-J. Guo, H.-M. Han, Jing-Wang, S.-J. Xiao, and Z.-D. Dai, "Surface-hydrophilic and protein-resistant silicone elastomers prepared by hydrosilylation of vinyl poly(ethylene glycol) on hydrosilanes-poly(dimethylsiloxane) surfaces," Colloids and Surfaces A: Physicochemical and Engineering Aspects, vol. 308, no. 1-3, pp. 129-135, 2007. 

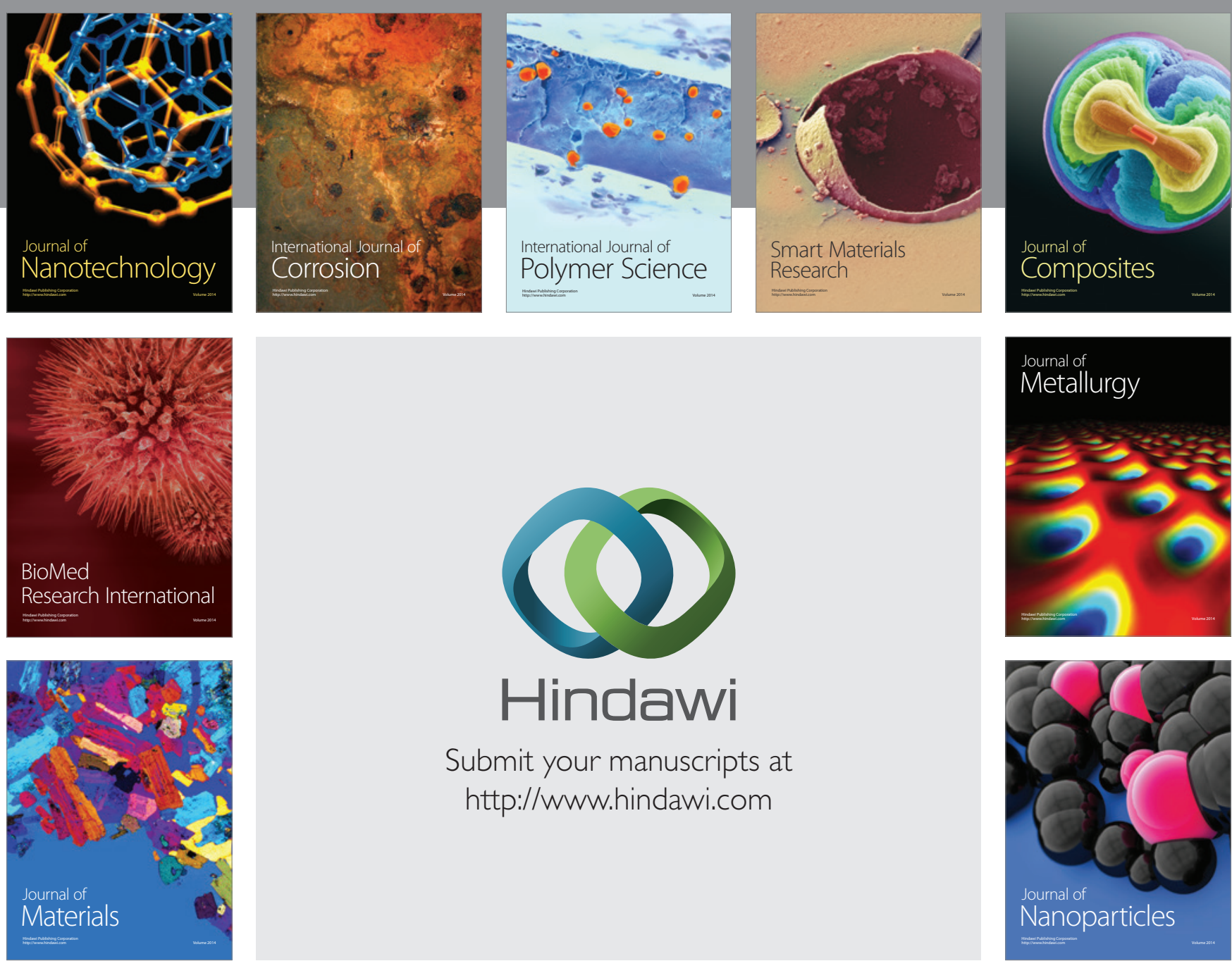

\section{Hindawi}

Submit your manuscripts at

http://www.hindawi.com

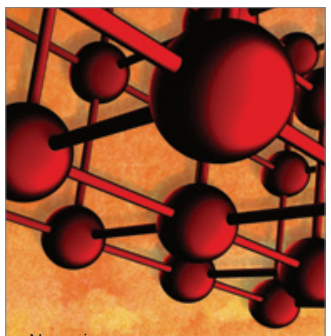

Materials Science and Engineering
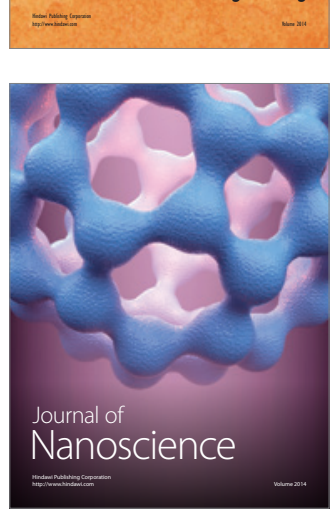
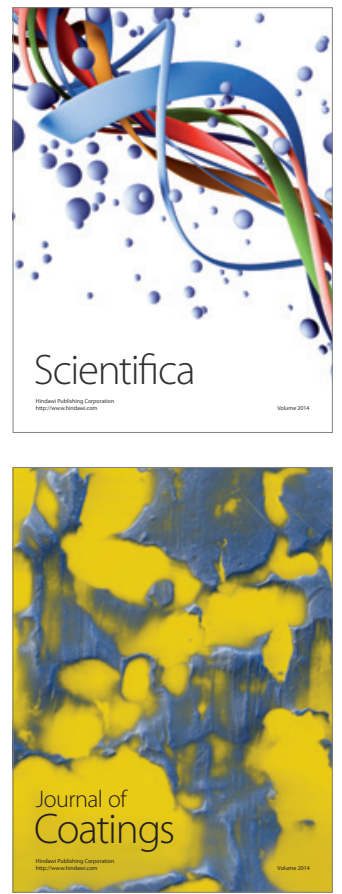
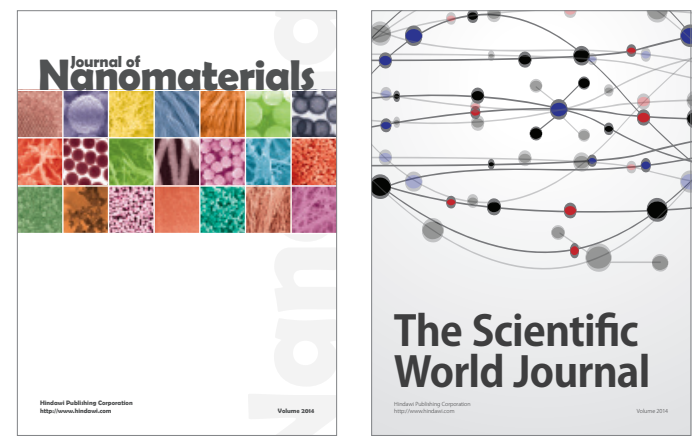

The Scientific World Journal
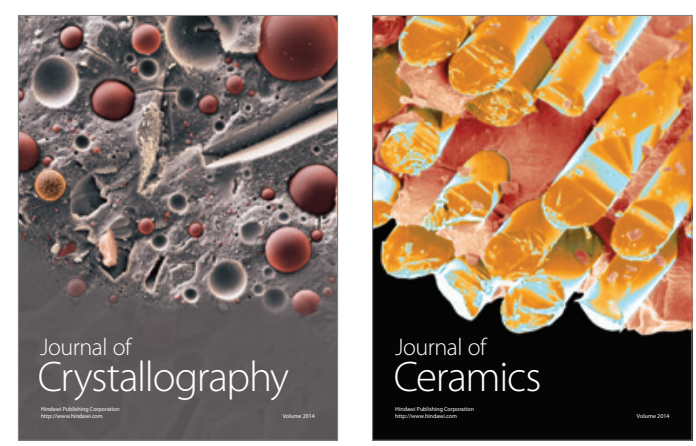
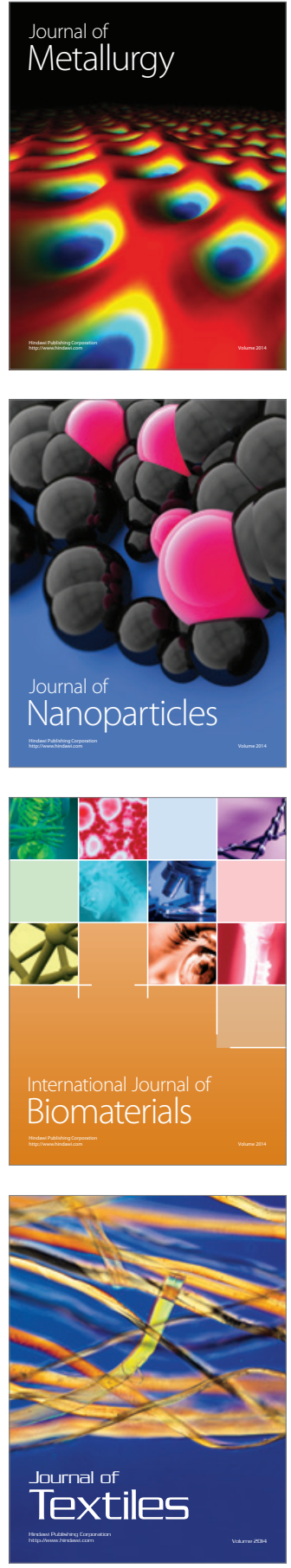